\title{
Geometric-phase-propagator approach to time-dependent quantum systems
}

\author{
F. K. Diakonos, ${ }^{1, *}$ P. A. Kalozoumis, ${ }^{1, \dagger}$ A. I. Karanikas, ${ }^{1, \dagger}$ N. Manifavas, ${ }^{1, \S}$ and P. Schmelcher ${ }^{2, \|}$ \\ ${ }^{1}$ Department of Physics, University of Athens, GR-15771 Athens, Greece \\ ${ }^{2}$ Zentrum fuer Optische Quantentechnologien, Universität Hamburg, Luruper Chaussee 149, 22761 Hamburg, Germany
}

(Received 12 March 2012; published 18 June 2012)

\begin{abstract}
A field-theoretical approach to the scattering off an oscillating quantum system is developed. As a key ingredient it employs the adiabatic eigenstate basis and consists of a perturbative scheme for the calculation of the geometric phases influencing the transmission through the time-dependent potential landscape. The main advantage is the identification of basic diagrams which allow for an immediate interpretation of the underlying elementary physical processes contributing to the scattering and transmission behavior. We apply our method to the simple, but prototypical, problem of transmission through an one-dimensional oscillating $\delta$ potential and demonstrate how it enables a deeper understanding of the relevant physical processes.
\end{abstract}

DOI: 10.1103/PhysRevA.85.062110

PACS number(s): 03.65.Db

\section{INTRODUCTION}

The transmission of quantum particles through a timedependent potential has been the subject of extensive studies in the last three decades (see Ref. [1] and references therein). The main goal in these studies is to classify and understand the mechanisms of quantum tunneling in such a potential. Typical examples of processes involving transmission through time-dependent devices are the tunneling of a test particle from a metastable state [2] using instanton techniques, scattering off a dissipative environment [3], and the tunneling through a timemodulated barrier [4]. Particularly, in Ref. [4] it was shown that, at low modulation frequencies, the traversing particle sees a static barrier and at high frequencies the particle tunnels through the time-averaged potential. It was also demonstrated that inelastic processes can occur, where the tunneling particle gains or loses energy quanta from the modulation field. In the same work a fundamental question was raised concerning the definition of the traversal time through such a fluctuating device, an issue which is still under debate [5]. Recently, novel perspectives for the study of driven quantum systems emerged. In particular, it has been realized that there is the possibility to exploit phenomena occurring in these systems for the development of novel technology such as the design of quantum pumps (i.e., devices capable to create quantum directed transport through periodic external driving [6]), the control of quantum devices for information processing [7], or the design of beam splitters for atom lasers [8,9].

One of the simplest examples for studying the transmission in a time-dependent environment is the tunneling through a $\delta$ barrier with a harmonically oscillating coupling, which has been considered by several authors [10-13]. Although the device is very simple the transmission coefficient possesses complicated structure, due to the presence of the timedependent coupling, containing Fano resonances, threshold enhancements related to sideband modes, and their interplay

\footnotetext{
*fdiakono@phys.uoa.gr

†pkalozoum@phys.uoa.gr

†akaran@phys.uoa.gr

§nmanif@phys.uoa.gr

"pschmelc@physnet.uni-hamburg.de
}

[11]. More complicated setups, involving several oscillating $\delta$ barriers, have been investigated in the context of quantum pumps [14]. In this case dephasing effects, which influence the appearance of resonances, may occur [15]. In [16] an infinite periodic chain of $\delta$ barriers with harmonically oscillating strength has been studied, demonstrating the modification of the conductance zones through the time dependence.

If the driving of the potential barrier is periodic, the standard approach used to solve the quantum dynamics is based on Floquet theory [17], which is very well suited for the straightforward calculation of the transmission properties, discriminating between the contributions from elastic and inelastic channels. In the Floquet treatment, the considered problem is first rendered time independent and then solved using standard numerical techniques. This certainly represents an advantage from the computational point of view. However, within this approach, a deeper physical insight requires additional, problem specific, efforts [18] and a general scheme for the identification of mechanisms leading to a particular dynamical behavior is still lacking.

Path integral methods, on the other hand side, have been commonly used for the study of the quantum dynamics in time-dependent potentials [19], but they show usually a slow convergence. Thus, despite of a few exceptional cases where an analytical solution can be obtained [20], there is no efficient general-purpose path integral treatment of time-dependent potentials. Progress in this direction has recently been achieved by introducing a rapidly converging scheme in the framework of a high-order short-time expansion of transition amplitudes in time-dependent potentials [21]. This approach holds for a general time dependence of the potential. The disadvantage of this approach is that it can only be applied to smooth potentials. This is a relevant point in view of the fact that previous works have mostly been employing either the $\delta$ or a rectangular barrier for the study of the time-dependent systems.

In the present work we develop an alternative theoretical approach to the investigation of the quantum dynamics in time-dependent potentials. The method is suitable for studying any kind of potentials (also nonsmooth) as well as external driving (periodic or not, smooth or nonsmooth). In addition it allows for a classification and decomposition of the quantum dynamics in terms of fundamental processes which represent the skeleton of the quantum evolution in these systems. 
Particularly, we reveal the role of virtual "multiphoton" processes which determine to a large extent details of the resonant structures in the dependence of the transmission coefficient on the incoming energy. Within our treatment these subprocesses can be clearly distinguished from the real "multiphoton" exchange which leads to inelastic transmission [4]. The power of our method is demonstrated using the example of the oscillating $\delta$ barrier, which has been extensively studied in the literature. We show that it is possible to calculate and understand transmission properties which have not been accessible so far. An example is the transmission zero associated with the Fano resonance characterizing the quantum dynamics in this system. The virtual "multiphoton" processes, mentioned above, are relevant for the determination of its location.

The paper is organized as follows: In Sec. II we present the main idea of the perturbative scheme used to describe the transmission properties of a time-dependent quantum device. In Sec. III we present our method focusing on the case of periodic driving. We investigate the $\delta$ barrier with a harmonically oscillating strength as a simple example. In Sec. IV we give the results for the transmission properties of the oscillating $\delta$-barrier setup. In particular, we demonstrate how one can systematically calculate and interpret details of the behaviour of the transmission coefficient as a function of the incoming energy within our approach. Section V contains our concluding remarks. Finally, extensive formulas and their derivation are provided in the appendices.

\section{IMPACT OF GEOMETRIC PHASES ON TRANSMISSION IN TIME-DEPENDENT POTENTIALS}

We consider the transmission of a quantum particle through a localized time-dependent potential. Our specific analysis is performed assuming a one-dimensional setup. However, the proposed approach can be easily generalized to higherdimensional cases. Initially (i.e., for $t \rightarrow-\infty$ ), the wave function of the quantum particle for $x \rightarrow-\infty$ is a plane wave with energy $E_{i}$ and momentum directed from the left to the right (incoming quantum particle). We also assume that the wave function for $t \rightarrow \infty$ and $x \rightarrow \infty$ is a plane wave with energy $E_{f}$ and the same direction of momentum (outgoing quantum particle). The amplitude for scattering of the considered quantum particle by a specific local timedependent potential is, in general, given in terms of the corresponding causal Green's function $\Delta_{n, m}$ as

$$
S_{f i}=-i \hbar e^{i\left(E_{f} t_{f}-E_{i} t_{i}\right) / \hbar} \sum_{n, m}\left\langle\vec{p}_{f} \mid n\left(t_{f}\right)\right\rangle \Delta_{n, m}\left(t_{f}, t_{i}\right)\left\langle m\left(t_{i}\right) \mid \vec{p}_{i}\right\rangle,
$$

where $t_{i}, t_{f}$ are the initial and final time, $\vec{p}_{i}$ and $\vec{p}_{f}$ are the input and output momenta of the particle, and $E_{i}$ and $E_{f}$ are the corresponding energies. In Eq. (1) we have introduced the temporary basis $\{|n(t)\rangle\}$ with $n$ being a collective index for all the quantum numbers needed to fully identify each temporary eigenstate of the time-dependent Hamiltonian $\hat{H}(t)$ at time $t$. In this basis the instantaneous Schrödinger equation reads:

$$
\hat{H}(t)|n(t)\rangle=E_{n}(t)|n(t)\rangle
$$

The relevant quantities for determining the Green's function $\Delta_{n, m}$ are the geometric phases [22] $\left\langle n(t)\left|i \hbar \partial_{t}\right| m(t)\right\rangle$ (for an introduction to geometric phases see Sec. 8.13 of Ref. [19]). The diagonal elements $\gamma_{n}(t) \equiv\left\langle n(t)\left|i \hbar \partial_{t}\right| n(t)\right\rangle$ are the Berry phases which are different from zero only when $u_{n}(x, t)=$ $\langle x \mid n(t)\rangle$ cannot be chosen real or, in other words, when the $E_{n}(t)$ level is degenerate. This is certainly the case when we are dealing with the continuum part of the spectrum and also for the bound part as long as we are working in more than one dimension. In our work we shall isolate the geometrical phases expressing transitions between states with different energy, for which it can be easily shown that

$$
\left\langle n(t)\left|i \hbar \partial_{t}\right| m(t)\right\rangle=\frac{\left\langle n(t)\left|i \hbar \partial_{t} \hat{H}(t)\right| m(t)\right\rangle}{E_{m}(t)-E_{n}(t)}, \quad\left[E_{m}(t) \neq E_{n}(t)\right] .
$$

In the temporary basis the propagating kernel $\Delta_{n, m}$ fulfils the Green's equation:

$\sum_{m}\left[\left(i \hbar \partial_{t}-\tilde{E}_{n}(t)\right) \delta_{n, m}-\Phi_{n, m}(t)\right] \Delta_{m, n^{\prime}}\left(t, t^{\prime}\right)=\delta_{n, n^{\prime}} \delta\left(t-t^{\prime}\right)$,

where the infinite-dimensional matrix $\Phi$ contains only the nondiagonal geometric phases:

$$
\Phi_{n, m}(t)=\left\langle n(t)\left|i \hbar \partial_{t}\right| m(t)\right\rangle-\delta_{n, m} \gamma_{m}(t),
$$

while the Berry phases are included in the effective energies $\tilde{E}_{n}(t)$ :

$$
\tilde{E}_{n}(t)=E_{n}(t)-\sum_{m} \delta_{n, m} \gamma_{m}(t)
$$

Using the completeness of the temporary eigenstates as well as the orthonormality relation $\left\langle n(t) \mid n^{\prime}\left(t^{\prime}\right)\right\rangle=\delta_{n, n^{\prime}} \delta\left(t-t^{\prime}\right)$ we can interpret (see, for example, Ref. [19]) the solution of Eq. (4) in operator form

$$
\Delta_{n, m}\left(t_{f}, t_{i}\right)=\left\langle n\left(t_{f}\right)\left|\frac{1}{\hat{H}_{0}-\tilde{E}-\hat{\Phi}}\right| m\left(t_{i}\right)\right\rangle, \quad \hat{H}_{0}=i \hbar \partial_{t} .
$$

The nondiagonal matrix $\Phi$ in Eq. (7) renders, in the general case, the considered problem analytically intractable. To proceed with the calculation of the amplitude (1) it is necessary to develop a scheme allowing for the expansion of the propagator in terms of simpler calculable subprocesses. The main assumption in our approach is that there exists an ordering with respect to the magnitude of the transition amplitudes of the different dynamical processes taking place in the scattering off a time-dependent potential. The latter is implied by the energy difference between the incoming and the outgoing state as stated in Eq. (3). The amplitudes of the elastic processes dominate while inelastic processes with small energy transfer are more probable than those with large energy transfer. This property, if valid and consistently applicable, suggests that the nondiagonal matrix $\Phi$ can be treated as a perturbation and the following expansion of $\Delta_{n, m}$ is possible:

$$
\Delta \equiv \frac{1}{\hat{H}_{0}-\tilde{E}-\hat{\Phi}}=\frac{1}{\hat{H}_{0}-\tilde{E}}+\frac{1}{\hat{H}_{0}-\tilde{E}} \hat{\Phi} \frac{1}{\hat{H}_{0}-\tilde{E}}+\cdots
$$


In Eq. (8) the expansion breaks down for zero eigenvalues of the denominator $\hat{H}_{0}-\tilde{E}$. Thus a special treatment is required in this case. We will come back to this point later on. Using the expansion (8) we can write the amplitude (1) as follows:

$$
\begin{aligned}
S_{f i}= & S_{f i, \text { static }}-i \hbar e^{i\left(E_{f} t_{f}-E_{i} t_{i}\right) / \hbar} \sum_{n, m}\left\langle\vec{p}_{f} \mid n\left(t_{f}\right)\right\rangle \\
& \times \sum_{r=1}^{\infty} \Delta_{n, m}^{(r)}\left(t_{f}, t_{i}\right)\left\langle m\left(t_{i}\right) \mid \vec{p}_{i}\right\rangle,
\end{aligned}
$$

where the $\Delta_{n, m}^{(i)}$ term in the above sum involves $i$ insertions of the transition matrix $\Phi$ while $S_{f i \text {,static }}$ is the zeroth-order term which does not contain $\hat{\Phi}$. The first term in the sum on the right-hand side (rhs) of Eq. (9) is

$$
\begin{aligned}
\Delta_{n, m}^{(1)}\left(t_{f}, t_{i}\right)= & \int_{-\infty}^{+\infty} d t_{1} \sum_{l_{1}, l_{2}}\left\langle n\left(t_{f}\right)\left|\frac{1}{\hat{H}_{0}-\tilde{E}}\right| l_{1}\left(t_{1}\right)\right\rangle \Phi_{l_{1}, l_{2}} \\
& \times\left\langle l_{2}\left(t_{1}\right)\left|\frac{1}{\hat{H}_{0}-\tilde{E}}\right| m\left(t_{i}\right)\right\rangle
\end{aligned}
$$

and the higher-order terms have a similar structure as implied by the expansion (8). The form (9) allows the use of a particle picture for the interpretation of the dynamics in scattering off a time-dependent potential having at the same time a simple diagrammatic interpretation: the amplitude $S_{f i}$ is decomposed into a sum of subprocesses. Each subprocess is a sequence of two elementary processes: the particle propagation being in a particular eigenstate and the transition between two states of the temporary basis. The explicit form of $S_{f i}$ depends on the applied potential. The power of the diagrammatic representation of $S_{f i}$ is that it allows the calculation of desired properties (like the energy of a transmission zero or a local transmission maximum) by isolating the contributing dynamical processes. In general the spectrum of $\hat{H}(t)$ contains both a discrete as well as a continuous part. Therefore, the allowed elementary processes can be classified as follows:

(i) continuum-continuum (c-c) transitions,

(ii) continuum-bound (c-b) and bound-continuum (b-c) transitions,

(iii) bound-bound (b-b) transitions,

(iv) propagation in a continuum state,

(v) propagation in the bound state.

A typical subprocess contributing to $S_{f i}$ is shown in Fig. 1. The curly line indicates propagation in a continuum state while the dashed line means propagation in a bound state. The full black circles indicate $\mathrm{c}-\mathrm{c}$ transitions while the circles containing a cross indicate a c-b or b-c transition.

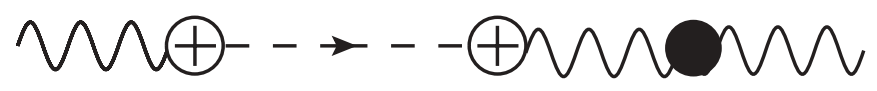

FIG. 1. Typical subprocess involving 3 transitions (continuumbound state, bound-state-continuum, and continuum-continuum) contributing to the transition amplitude (1) at the third order of the proposed perturbation scheme.

\section{CASE OF TIME-PERIODIC POTENTIALS: A $\delta$-BARRIER WITH OSCILLATING STRENGTH}

The calculation of the transmission properties in a timedependent potential will significantly simplify if the driving is periodic. In this section we will demonstrate how the perturbative scheme introduced in the previous section works in practice by performing an analysis of the transmission properties of a monochromatic wave passing through a periodically varying potential. As a concrete example we consider the transmission through a $\delta$ barrier with oscillating strength in one dimension. As mentioned already in the introduction, our main purpose is to calculate and explain features of the transmission behavior which are not easily accessible by other approaches like direct integration or Floquet theory. The Schrödinger equation of the considered problem reads

$$
\begin{aligned}
i \hbar \frac{\partial \Psi(x, t)}{\partial t} & =-\frac{\hbar^{2}}{2 m} \frac{\partial^{2} \Psi(x, t)}{\partial x^{2}}-g(t) \delta(x) \Psi(x, t), \\
g(t) & =g(t+T),
\end{aligned}
$$

with $T=2 \pi / \omega$ being the period of the oscillating $\delta$ potential and $\omega$ being the associated frequency. For fixed $t$ the problem becomes static and can be easily solved. Introducing the length scale $\ell_{0}=\sqrt{\hbar /(m \omega)}$ and the energy scale $\epsilon_{0}=\hbar \omega$ the static version of Eq. (11) can be written in dimensionless form as follows:

$$
\left(-\frac{1}{2} \frac{d^{2}}{d \xi^{2}}-g_{\tau} \delta(\xi)\right) u\left(\xi ; g_{\tau}\right)=\epsilon(\tau) u\left(\xi ; g_{\tau}\right), \quad \tau=\omega t,
$$

with $\xi=x / \ell_{0}, g_{\tau}=\frac{1}{\hbar \omega} \sqrt{m \omega / \hbar} g(\tau), \epsilon(\tau)=E(\tau) / \epsilon_{0}$, and $u\left(\xi ; g_{\tau}\right)$ is the corresponding wave function. For simplicity we assume here $g(\tau)=g_{0} \sin (\tau)$ with strength constant $g_{0}$. In this case, for a given time instant, the spectrum of the Hamiltonian consists of continuum states and one bound state with energy $\epsilon_{b}=-\frac{1}{2} g_{\tau}^{2}$. The latter exists only during the time period for which $\sin (\tau)>0$. The associated complete and orthonormal temporary basis is

$$
\begin{aligned}
u_{k}^{ \pm}\left(\xi ; g_{\tau}\right) & =\frac{1}{\sqrt{2 \pi}}\left(e^{ \pm i k \xi}-\frac{g_{\tau}}{g_{\tau}+i k} e^{i k|\xi|}\right), \quad k=\sqrt{2 \epsilon}, \\
\epsilon & >0, \quad u_{b}\left(\xi ; g_{\tau}\right)=\sqrt{g_{\tau}} e^{-g_{\tau}|\xi|}, \quad g_{\tau}>0,
\end{aligned}
$$

as given in Ref. [23].

The perturbative calculation of the scattering amplitude Eq. (1) requires the summation of all the contributing subprocesses in increasing order. According to the discussion in the previous section the order of a term in the perturbation series is determined by the number of transitions (c-c, b-c, or c-b) and can be diagrammatically presented by the series of graphs shown up to second order in Fig. 2. Since there is only one bound state in the temporary spectrum there are no possible transitions between bound states.

To first order only processes with a single transition, necessarily of $\mathrm{c}-\mathrm{c}$ type, participate. These may be elastic or inelastic. In the inelastic case the final energy is given as $\epsilon_{f}=\sqrt{\epsilon_{i}^{2}+n}$ where $n$ takes the values $n= \pm 1, \pm 2, \ldots$ under the restriction that $\epsilon_{i}^{2}+n$ remains positive. This is a consequence of the periodic driving [4] valid for all orders of the proposed perturbation expansion (real "multiphoton" 


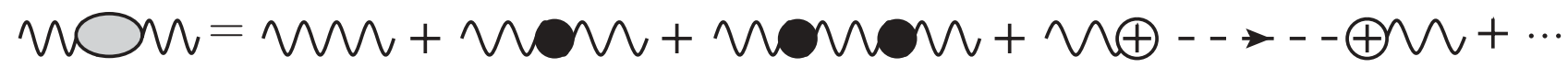

FIG. 2. Diagrammatic expansion for amplitude (1) up to second order in the introduced perturbative scheme.

exchange). Before going on with the explicit calculation of the transmission amplitude (1) it is useful to present the matrix elements for the building blocks of the contributing subprocesses.

Continuum-continuum transitions. The transition amplitude from one continuum state to another has the form

$$
\begin{aligned}
\Phi_{k k^{\prime}}(\tau) & \equiv \frac{1}{\hbar \omega}\left\langle k^{( \pm)}(\tau)\left|i \hbar \partial_{\tau}\right| k^{\prime( \pm)}(\tau)\right\rangle \\
& =\frac{i}{\pi} \dot{g}_{\tau} \frac{e^{i\left[\theta_{k^{\prime}}(\tau)-\theta_{k}(\tau)\right]}}{\left(g_{\tau}^{2}+k^{2}\right)^{1 / 2}\left(g_{\tau}^{2}+k^{\prime 2}\right)^{1 / 2}} \frac{k k^{\prime}}{k^{2}-\left(k^{\prime}+i \eta\right)^{2}}
\end{aligned}
$$

with $\theta_{k}(\tau)=\arctan \left(g_{\tau} / k\right)$. The superscript $( \pm)$ is omitted in $\Phi_{k k^{\prime}}(\tau)$ since the rhs of Eq. (15) is independent of these signs. As expected, the diagonal term $k=k^{\prime}$ diverges and needs to be regularized. We adopt the usual box regularization in order to define a regularized Berry phase:

$$
\begin{aligned}
\left\langle k^{( \pm)}(\tau)\left|i \hbar \partial_{\tau}\right| k^{( \pm)}(\tau)\right\rangle_{\text {reg }} & \equiv \frac{2 \pi}{V}\left\langle k^{( \pm)}(\tau)\left|i \hbar \partial_{\tau}\right| k^{( \pm)}(\tau)\right\rangle \\
& =\frac{1}{\hbar \omega} \gamma_{k}(\tau) \\
& =-\frac{1}{\hbar \omega} \dot{g}_{\tau} \frac{k}{g_{\tau}^{2}+k^{2}}
\end{aligned}
$$

where $\frac{V}{2 \pi}=\int_{V} \frac{d \xi}{2 \pi}=\delta_{R}(0)$ with $\delta_{R}(0)$ being the $\delta$ function regularized on a finite volume $V$. With this choice $\eta$ in Eq. (14) is finite, fulfilling $\eta V \propto 1$. Including also the off-diagonal terms we get the general, regularized expression, for $\Phi_{k k^{\prime}}(\tau)$ :

$$
\Phi_{k k^{\prime}}(\tau)=\frac{1}{\hbar \omega}\left[\left\langle k^{( \pm)}(\tau)\left|i \hbar \partial_{\tau}\right| k^{\prime( \pm)}(\tau)\right\rangle-\gamma_{k}(\tau) \delta\left(k-k^{\prime}\right)\right],
$$

for which it can be deduced that $\delta\left(k-k^{\prime}\right) \Phi_{k k^{\prime}}(\tau)=0$.

Continuum-bound-state transitions. Denoting $|b(\tau)\rangle$ the unique bound state of the potential (which exists only for $\tau \in(2 \pi m, 2 \pi m+\pi)$ with $m=0, \pm 1, \pm 2, \ldots)$ we can express the $\mathrm{c}-\mathrm{b}$ transition as

$$
\begin{aligned}
\Phi_{b k}(\tau) & =\frac{1}{\hbar \omega}\left\langle b(\tau)\left|i \hbar \partial_{\tau}\right| k^{( \pm)}(\tau)\right\rangle \\
& =-2 i k \sqrt{\frac{g_{\tau}}{2 \pi}} \frac{\dot{g}_{\tau}}{\left(g_{\tau}^{2}+k^{2}\right)^{3 / 2}} e^{i \theta_{k}(\tau)} .
\end{aligned}
$$

Obviously, the b-c transition amplitude $\Phi_{k b}$ is the complex conjugate of $\Phi_{b k}$.

The zero-order approximation of the scattering amplitude (1) is given as

$$
\begin{aligned}
S_{f i}^{(0)}= & -i e^{i\left(\epsilon_{f} \tau_{f}-\epsilon_{i} \tau_{i}\right)}\left\{\Delta_{b}^{(0)}\left(\tau_{f}, \tau_{i}\right)\left\langle k_{f} \mid b\right\rangle\left\langle b \mid k_{i}\right\rangle-\int_{0}^{\infty} d k \Delta_{k}^{(0)}\right. \\
& \left.\times\left(\tau_{f}, \tau_{i}\right)\left[\left\langle k_{f} \mid k^{(+)}\left(\tau_{f}\right)\right\rangle\left\langle k^{(+)}\left(\tau_{i}\right) \mid k_{i}\right\rangle+(+\leftrightarrow-)\right]\right\} .
\end{aligned}
$$

In Eq. (18) $\Delta_{b}^{(0)}$ is the propagator in the bound state while $\Delta_{k}^{(0)}$ is the propagator in a continuum state. The causal form of the latter reads:

$$
\Delta_{k}^{(0)}\left(\tau_{f}, \tau_{i}\right)=i \theta\left(\tau_{f}-\tau_{i}\right) \exp \left\{-i \int_{\tau_{i}}^{\tau_{f}} d \tau_{1}\left[\epsilon_{k}-2 \gamma_{k}\left(\tau_{1}\right)\right]\right\} .
$$

The bound-state propagator is, in the interval $0<\tau, \tau^{\prime}<2 \pi$, the solution of the Green's equation

$$
\left[i \partial_{\tau}-\epsilon_{b}(\tau)\right] \Delta_{b}^{(0)}\left(\tau, \tau^{\prime}\right)=-\delta\left(\tau-\tau^{\prime}\right) .
$$

The causal solution of Eq. (20) is

$$
\Delta_{b}^{(0)}\left(\tau, \tau^{\prime}\right)=i \theta\left(\tau-\tau^{\prime}\right) \exp \left[-i \int_{\tau^{\prime}}^{\tau} d \tau_{1} \epsilon_{b}\left(\tau_{1}\right)\right],
$$

while the solution obeying the periodic boundary condition $\Delta_{b}^{(0)}\left(\tau, \tau^{\prime}\right)=\Delta_{b}^{(0)}\left(\tau+2 \pi n, \tau^{\prime}\right)(n=0, \pm 1, \pm 2, \ldots)$ reads

$$
\begin{aligned}
\Delta_{b}^{(0)}\left(\tau, \tau^{\prime}\right)= & i \sum_{m=-\infty}^{\infty} \theta\left(\tau-\tau^{\prime}-2 \pi m\right) \\
& \times \exp \left[-i \int_{\tau^{\prime}+2 \pi m}^{\tau} d \tau_{1} \epsilon_{b}\left(\tau_{1}\right)\right] .
\end{aligned}
$$

To obtain the $S$-matrix amplitude one has to perform the limits $\tau_{i} \rightarrow-\infty, \tau_{f} \rightarrow \infty$. Thus a consistent treatment requires $\tau_{f}=-\tau_{i}=\lim _{N \rightarrow \infty} \frac{N \pi}{\omega}$ such that $g\left(\tau_{f}\right)=g\left(\tau_{i}\right)=0$. In this case the zero-order contribution to the scattering amplitude is trivial containing no transitions (free transmission). We will use in the following the notation $(n t, m c, l b)$ for the classification of the contribution of the various subprocesses to the $S$ matrix. $n t$ means that the considered subprocess contains in total $n$ transitions. From these $n$ transitions $m$ are of c-c type while $l$ are of b-c or c-b type. Using this notation we write

$$
S_{f i}^{(0 t, 0 c, 0 b)}=\delta\left(k_{f}-k_{i}\right) .
$$

The first-order term, containing a single c-c transition, becomes

$$
\begin{aligned}
S_{f i}^{(1 t, 1 c, 0 b)}= & -i e^{i\left(\epsilon_{f} \tau_{f}-\epsilon_{i} \tau_{i}\right)} \int_{-\infty}^{\infty} d \tau_{1} \Delta_{k_{f}}^{(0)}\left(\tau_{f}, \tau_{1}\right) \Phi_{k_{f} k_{i}} \\
& \times\left(\tau_{1}\right) \Delta_{k_{i}}^{(0)}\left(\tau_{1}, \tau_{i}\right) .
\end{aligned}
$$

Obviously, there are no first-order processes involving a single $b-c$ or $c-b$ transition since the outgoing and incoming states belong necessarily to the continuum spectrum (positive energy). Equation (24) can be rewritten as

$$
\begin{aligned}
S_{f i}^{(1 t, 1 c, 0 b)}= & 2 \pi i \sum_{n \neq 0} \frac{A_{k_{f} k_{i}}(n)}{\left|k_{f}\right|} \delta\left(k_{f}-\sqrt{k_{i}^{2}+2 n}\right) \\
& +\left(k_{f} \rightarrow-k_{f}\right),
\end{aligned}
$$

with

$$
e^{2 i \theta_{k_{f}}(\tau)} \Phi_{k_{f} k_{i}} e^{-2 i \theta_{k_{i}}(\tau)}=\sum_{n=-\infty}^{\infty} A_{k_{f} k_{i}}(n) e^{-i n \tau}
$$


and therefore

$$
A_{k_{f} k_{i}}(n)=\int_{0}^{2 \pi} \frac{d \tau}{2 \pi}\left[e^{2 i \theta_{k_{f}}(\tau)} \Phi_{k_{f} k_{i}} e^{-2 i \theta_{k_{i}}(\tau)}\right] e^{i n \tau} .
$$

Since the renormalized c-c transition obeys

$$
\Phi_{k_{f} k_{i}} \delta\left(k_{f}-k_{i}\right)=0,
$$

the term $n=0$ is not included in the sum of Eq. (25). The calculation of the amplitude $A_{k_{f} k_{i}}$, introduced to describe the $S$-matrix contribution of the c-c transitions is straightforward and leads to the expression

$$
\begin{aligned}
A_{k_{f} k_{i}}(n) & =\frac{i}{\pi} \frac{k_{f} k_{i}}{k_{f}^{2}-k_{i}^{2}} \frac{1}{k_{f}+k_{i}}\left[q_{k_{i}}(|n|)-(-1)^{n} q_{k_{f}}(|n|)\right] s(n), \\
s(n) & =\theta(n)-(-1)^{n} \theta(-n), \\
q_{k}(n) & =\frac{1}{g_{0}^{n}}\left(\sqrt{k^{2}+g_{0}^{2}}-k\right)^{n},
\end{aligned}
$$

with $A_{k_{f} k_{i}}(0)=0$. The various $n$ contributing to the sum in Eq. (25) correspond to the usual higher Floquet modes. As it can be seen from Eq. (28) the amplitude $A_{k_{f} k_{i}}$ for the inelastic continuum-continuum transitions decays exponentially with $|n|$ for $g_{0} / k_{<}<1$ [where $k_{<}=\min \left(k_{f}, k_{i}\right)$ ]:

$$
A_{k_{f} k_{i}}(n) \underset{g_{0} \rightarrow 0}{\approx}\left(\frac{g_{0}}{k_{<}}\right)^{|n|}
$$

in accordance with the fast convergence of the Floquet sum observed in the analysis of the dynamics of the oscillating $\delta$ barrier in the literature [24]. Notice that, for $g_{0} / k_{<}>1$, although the approximation (28) does not hold, the perturbative expansion is still valid. The reason is that, also in this case, the contribution of the diagrams with increasing number of transitions decreases. Since the dimensionless coupling $g_{0}$ is given by the original coupling multiplied by a factor proportional to $\omega^{-1 / 2}$ our treatment is necessarily nonadiabatic and becomes exact either in the weak-coupling or in the rapid-oscillation (very large oscillation frequency $\omega$ ) limit. One important issue to be noticed here is that the expansion of the $S$ matrix in terms of the number of transitions, as described above, allows the decomposition of the transmission process into elementary subprocesses giving a consistent meaning to our approximation procedure. The emerging perturbative scheme can also be understood in terms of an expansion in powers of the ratio $g_{0} / k_{i}$ of the coupling $g_{0}$ over magnitude of the incoming wave vector $k_{i}$. As can be directly confirmed from Eqs. (16) and (17), the c-c transitions are of order $g_{0} / k_{i}$ while $\mathrm{c}-\mathrm{b}$ or b-c transitions are of order $\left(g_{0} / k_{i}\right)^{3 / 2}$. The magnitude of the various diagrammatic contributions to the $S$ matrix is then quantified by the leading power of $g_{0} / k_{i}$, a power that increases as the number of transitions increases.

In the next order (two transitions) the c-b or b-c transitions are also possible. Typically, the corresponding subprocess is demonstrated by the fourth diagram on the rhs shown in Fig. 2. The contribution of this term to the amplitude $S_{f i}$ is given as

$$
\begin{aligned}
S_{f i}^{(2 t, 0 c, 2 b)}= & i \int_{-\infty}^{\infty} d \tau_{2} \int_{-\infty}^{\tau_{2}} d \tau_{1} e^{i\left(\epsilon_{f} \tau_{2}-\epsilon_{i} \tau_{1}\right)} e^{2 i \theta_{k_{f}}\left(\tau_{2}\right)} \Phi_{k_{f} b}\left(\tau_{2}\right) \\
& \times \Delta_{b}^{(0)}\left(\tau_{2}, \tau_{1}\right) \Phi_{b k_{i}}\left(\tau_{1}\right) e^{-2 i \theta_{k_{i}}\left(\tau_{1}\right)} \theta\left(k_{f}\right)
\end{aligned}
$$

This amplitude is nonzero only if the time variables are in the region $(2 \pi \nu, \pi+2 \pi \nu)$. The needed bound-state propagator fulfils the periodic boundary condition $\Delta_{b}^{(0)}\left(\tau_{2}, \tau_{1}\right)=$ $\Delta_{b}^{(0)}\left(\tau_{2}+2 \pi m, \tau_{1}\right)(m=0, \pm 1, \ldots)$ and is given by Eq. (22) where the allowed number of terms which must be summed up depends on the difference $\tau_{2}-\tau_{1}$. These facts complicate the calculation of the amplitude (29). We can considerably simplify things by replacing the time-dependent bound-state energy, in the framework of our approximation scheme, by its mean value over a period:

$$
\bar{\epsilon}_{b}=\frac{1}{2 \pi} \int_{0}^{2 \pi} d \tau \epsilon_{b}(\tau)=-\frac{1}{8} g_{0}^{2} .
$$

This approximation is a first-order estimation, independent of the specific form of the oscillating potential, being justified in the regime of very fast or very slow (static limit) oscillations of the potential which lead to an effective, time-independent, bound-state energy. Higher-order corrections can be obtained, in our case, by expanding the bound-state wave function around the effective coupling value $g_{0} / 2$. Following this scheme the b-c transition amplitude becomes, in first order,

$$
\bar{\Phi}_{k b}(\tau)=2 i k \sqrt{\frac{g_{0}}{4 \pi}} \frac{\dot{g}_{\tau}}{k-i g_{0} / 2} \frac{e^{-2 i \theta_{k}(\tau)}}{k^{2}+g_{\tau}^{2}} .
$$

Introducing now the Fourier transformations:

$$
\begin{aligned}
e^{2 i \theta_{k_{f}}(\tau)} \bar{\Phi}_{k_{f} b}(\tau) & =\sum_{n=-\infty}^{\infty} B_{k_{f} b}(n) e^{-i n \tau}, \\
\bar{\Phi}_{b k_{i}}(\tau) e^{-2 i \theta_{k_{i}}(\tau)} & =\sum_{n=-\infty}^{\infty} B_{b k_{i}}(n) e^{-i n \tau},
\end{aligned}
$$

with

$$
\begin{aligned}
& B_{k_{f} b}(n)=\frac{1}{2 \pi} \int_{0}^{2 \pi} d \tau e^{2 i \theta_{k_{f}}(\tau)} \bar{\Phi}_{k_{f} b}(\tau) e^{i n \tau}, \\
& B_{b k_{i}}(n)=\frac{1}{2 \pi} \int_{0}^{2 \pi} d \tau \bar{\Phi}_{b k_{i}}(\tau) e^{-2 i \theta_{k_{i}}(\tau)} e^{i n \tau},
\end{aligned}
$$

we obtain for the amplitude $B_{k b}(n)$,

$$
B_{k b}(n)=i \sqrt{\frac{g_{0}}{4 \pi}} \frac{1}{k-i g_{0} / 2} q_{k}(|n|)\left[1-(-1)^{n}\right]=B_{b k}^{*}(-n),
$$

where $q_{k}(n)$ is given by Eq. (27). It is straightforward to show that also the b-c transition amplitudes $B_{k b}(n)$ decay exponentially with the Floquet index $n$ when the applied perturbation scheme is valid; namely,

$$
\left|B_{k b}(n)\right|_{g_{0} \rightarrow 0}^{\approx} \frac{1}{\sqrt{k}}\left(\frac{g_{0}}{k}\right)^{|n|+\frac{1}{2}} .
$$

Using the expressions (32) we can calculate the contribution of the $c-b$ or $b-c$ transitions corresponding to the third diagram on the right-hand side of Fig. 2 to the $S$ matrix as follows:

$$
\begin{aligned}
S_{f i}^{(2 t, 0 c, 2 b)}= & -2 \pi i \sum_{n=-\infty}^{\infty} \frac{B_{k_{f} k_{i}}(n)}{\left|k_{f}\right|} \theta\left(k_{f}\right) \delta\left(k_{f}-\sqrt{k_{i}^{2}+2 n}\right) \\
& +\left(k_{f} \leftrightarrow-k_{f}\right)
\end{aligned}
$$


where

$$
B_{k_{f} k_{i}}(n)=\sum_{n_{0}=-\infty}^{\infty} \frac{B_{k_{f} b}\left(n+n_{0}\right) B_{b k_{i}}\left(-n_{0}\right)}{\tilde{\epsilon}_{i}-n_{0}+i \eta},
$$

and $\tilde{\epsilon}_{i}=\epsilon_{i}-\bar{\epsilon}_{b}$. The small parameter $\eta$ in the denominator of (37) is introduced in order to ensure convergence for $\tau_{i} \rightarrow-\infty$ and is equivalent to the demand that the continuum Green's functions vanish in this limit. In the absence of $\eta$ the amplitude $B_{k_{f} k_{i}}$ possesses a pole at $\tilde{\epsilon}_{i}=n_{0}$. The occurrence of the pole is, from a mathematical point of view, a result of the perturbative expansion. In fact, the time dependence of the potential produces an effective Hamiltonian containing an infinite series of geometric phases [22]. These terms are the origin of a nonvanishing imaginary part $\eta^{R}$ that naturally appears when higher-order terms, involving virtual transitions between the continuum and the bound state (virtual "multiphoton" exchange), are summed up. As a consequence, the denominator of the amplitude (37) never vanishes on the real axis of the energy. At the same time these higher-order terms shift the energy $\tilde{\epsilon}_{i}$ by $\delta \epsilon$. This is equivalent to a change in the effective bound-state energy of the form

$$
\bar{\epsilon}_{b} \rightarrow \bar{\epsilon}_{b}^{R}=-\frac{1}{8} g_{0}^{2}-\delta \epsilon .
$$

Including the higher-order corrections in (37) we obtain a normalized redefinition of the continuum-bound-continuum (c-b-c) transition:

$$
B_{k_{f} k_{i}}(n) \rightarrow B_{k_{f} k_{i}}^{R}(n)=\sum_{n_{0}=-\infty}^{\infty} \frac{B_{k_{f} b}\left(n+n_{0}\right) B_{b k_{i}}\left(-n_{0}\right)}{\tilde{\epsilon}_{i}^{R}-n_{0}+i \eta_{n}^{R}} Z_{n},
$$

where $Z_{n}$ is a normalization factor and $\tilde{\epsilon}_{i}^{R}, \eta^{R}$ are the corrected values. In Appendix A we present the detailed calculation of the renormalized factors indicating the significance of the virtual "multiphoton" exchange processes. Here, it suffices to note that, in the limit $g_{0} / k_{i}<1$ we have $B_{k_{f} b}\left(n+n_{0}\right) B_{b k_{i}}\left(-n_{0}\right) \sim O\left(g_{0}^{\left|n+n_{0}\right|+\left|n_{0}\right|+1}\right)$ which in turn means that, at least, $B_{k_{f} k_{i}} \sim O\left(g_{0}^{3}\right)$ (for $n=0, n_{0}= \pm 1$, or $n=n_{0}=-1$ ). On the other hand, the behavior of the corrected factors in the denominator of (39) depends only weakly on $n$ and $n_{0}$ [see Eq. (A4) and the discussion below this equation in Appendix A]: $\bar{\epsilon}_{b}^{R} \sim O\left(g_{0}^{2}\right), \eta^{R} \sim O\left(g_{0}^{3}\right)$. As a consequence, the corrected values play an essential role only when the incoming energy is close to an integer value.

The expression (39) is finite and well behaved for all the values of the incoming energy. However, when the energy of the incoming particle differs from the effective bound-state energy by a positive integer, the probability amplitude to arrive at the final state passing through the bound state has a sharp maximum. The impact of this maximum on the transmission properties will be discussed below. To complete the calculation of the $S$ matrix with two transitions we have to calculate also the contribution of the second diagram on the rhs of Fig. 2 containing two c-c transitions. After some straightforward steps we obtain

$$
S_{f i}^{(2 t, 2 c, 0 b)}=-4 \pi i \sum_{n=-\infty}^{\infty} \Gamma_{k_{f} k_{i}}(n) \frac{\theta\left(k_{f}\right)}{\left|k_{f}\right|} \delta\left(k_{f}-\sqrt{k_{i}^{2}+2 n}\right),
$$

with

$$
\Gamma_{k_{f} k_{i}}(n)=\int_{0}^{\infty} d k \sum_{l=-\infty}^{\infty} \frac{A_{k_{f} k}(n-l) A_{k k_{i}}(l)}{\epsilon_{i}-\epsilon_{k}+l+i 0^{+}}
$$

and $A_{k k^{\prime}}$ given by Eq. (27). Due to the integration over $k$ the regularizing imaginary part in the denominator of Eq. (41) can be taken as zero. It can be further confirmed, both numerically and analytically (see Appendix A), that the imaginary part of the above amplitude

$$
\begin{aligned}
\operatorname{Im}_{k_{f} k_{i}}(n) & =-\pi \sum_{\substack{l=-\infty \\
k_{i}^{2}+2 l \geqslant 0}}^{\infty} \frac{1}{\left|k_{l}\right|} A_{k_{f} k_{l}}(n-l) A_{k_{l} k_{i}}(l), \\
k_{l} & =\sqrt{k_{i}^{2}+2 l},
\end{aligned}
$$

which is controlled by the pole at $\epsilon_{k}=\epsilon_{i}+l$ dominates over its real part

$$
\operatorname{Re} \Gamma_{k_{f} k_{i}}(n)=\operatorname{Pr} \int_{0}^{\infty} d k \sum_{l=-\infty}^{\infty} \frac{A_{k_{f} k}(n-l) A_{k k_{i}}(l)}{\epsilon_{i}-\epsilon_{k}+l}
$$

that avoids the pole ( $\operatorname{Pr}$ denotes the principal value integration). In the case $g_{0} / k_{i}<1$, the imaginary part is of order $O\left(g_{0}^{|n-l|+|l|}\right)$ which, for $n=0, l= \pm 1$, is of order $O\left(g_{0}^{2}\right)$, while the real part is, at least, of order $O\left(g_{0}^{4}\right)$.

Already at this general stage of the analysis one can gain some insight into the expected behavior of the transmission coefficient. Whenever a propagator is contained in a diagram contributing to the transmission amplitude we observe the appearance of denominators which are in fact associated with the $1 /\left(\hat{H}_{0}-\tilde{E}\right)$ factors occurring in the expansion (10). These denominators are mainly responsible for resonant structures in the transmission coefficient. The most important contributions come from diagrams involving propagators close to the incoming and outgoing states since in this case a pole structure in the complex energy plane emerges. If the propagators are bracketed between transitions which do not involve the incoming or the outgoing state then the pole structure is integrated out leading to a smoother contribution to the transmission coefficient. However, the effect of these denominators is also controlled by the transition amplitudes which occur in the numerator and play the role of residues in the final expression for the contribution of a given diagram. This interplay between propagation and transition (poles and residues) determines the overall behavior of the transmission coefficient.

\section{TRANSMISSION PROPERTIES}

Having calculated the amplitudes of all subprocesses involving up to two transitions it is straightforward to calculate the associated $S$-matrix amplitude up to this order. In general, for scattering off a potential varying periodically with time, the $S$-matrix amplitude is given as

$$
\begin{aligned}
S_{f i}= & T_{i i}(0) \delta\left(k_{f}-k_{i}\right)+R_{i i}(0) \delta\left(k_{f}+k_{i}\right) \\
& +\sum_{n \neq 0}\left[T_{f i}(n) \delta\left(k_{f}-\sqrt{k_{i}^{2}+2 n}\right)\right. \\
& \left.+R_{f i}(n) \delta\left(k_{f}+\sqrt{k_{i}^{2}+2 n}\right)\right],
\end{aligned}
$$


where $T_{i i}(0)$ and $T_{f i}(n)$ are, respectively, the elastic and inelastic transmission amplitudes while $R_{i i}(0)$ and $R_{f i}(n)$ are the corresponding reflection amplitudes. They can be expressed in terms of the calculated subprocesses as follows:

$$
\begin{aligned}
T_{i i}(0)= & 1-\frac{2 \pi i}{\left|k_{i}\right|} B_{k_{i} k_{i}}^{R}(0)-\frac{4 \pi i}{\left|k_{i}\right|} \Gamma_{k_{i} k_{i}}(0)+\cdots, \\
R_{i i}(0)= & -\frac{2 \pi i}{\left|k_{i}\right|} B_{-k_{i} k_{i}}^{R}(0)-\frac{4 \pi i}{\left|k_{i}\right|} \Gamma_{-k_{i} k_{i}}(0)+\cdots, \\
T_{f i}(n)= & \frac{2 \pi i}{\left|k_{f}\right|} A_{k_{f} k_{i}}(n)-\frac{2 \pi i}{\left|k_{f}\right|} B_{k_{f} k_{i}}^{R}(n)-\frac{4 \pi i}{\left|k_{f}\right|} \Gamma_{k_{f} k_{i}}(n) \\
& +\cdots \quad(n \neq 0), \\
R_{f i}(n)= & \frac{2 \pi i}{\left|k_{f}\right|} A_{-k_{f} k_{i}}(n)-\frac{2 \pi i}{\left|k_{f}\right|} B_{-k_{f} k_{i}}^{R}(n)-\frac{4 \pi i}{\left|k_{f}\right|} \Gamma_{-k_{f} k_{i}}(n) \\
& +\cdots \quad(n \neq 0) .
\end{aligned}
$$

The total transmission coefficient is obtained as

$$
T_{\text {tot }}\left(\epsilon_{i}\right)=\left|T_{i i}(0)\right|^{2}+\sum_{\substack{n=-\infty \\ n \neq 0}}^{\infty} \frac{\left|k_{f}(n)\right|}{k_{i}}\left|T_{f i}(n)\right|^{2} .
$$

Notice that, in Eq. (43), we do not include interference terms which are of higher order in the proposed perturbative scheme. Of course such terms have to be taken into account in higherorder calculations.

Contribution of continuum-continuum versus continuumbound-state (or $b$-c) transitions. The decomposition (43) allows us to isolate the contribution to the transmission of the subprocesses involving $\mathrm{c}-\mathrm{b}$ or $\mathrm{b}-\mathrm{c}$ transitions and to compare with the corresponding contribution of subprocesses involving exclusively c-c transitions. This is not possible in any other approach. In addition, as in Floquet theory, we can also here discriminate between elastic and inelastic contributions to the transmission. Let us first concentrate on the dominating elastic transmission channel. According to Eq. (43) the elastic part of the transmission coefficient is given as

$$
\begin{aligned}
T_{i i}(0)= & 1-\frac{2 \pi i}{\left|k_{i}\right|} B_{k_{i} k_{i}}^{R}(0)-\frac{4 \pi i}{\left|k_{i}\right|} \Gamma_{k_{i} k_{i}}(0)+\cdots \\
= & 1-\frac{2 \pi i}{\left|k_{i}\right|} \sum_{n_{0}} \frac{B_{k_{i} b}\left(n_{0}\right) B_{b k_{i}}\left(-n_{0}\right)}{\tilde{\epsilon}_{i}^{R}\left(n_{0}\right)-n_{0}+i \eta_{0}^{R}\left(n_{0}\right)} Z_{0} \\
& -\frac{4 \pi i}{\left|k_{i}\right|} \Gamma_{k_{i} k_{i}}(0)+\cdots .
\end{aligned}
$$

The discussion below Eqs. (39) and (41) indicates that the dominant contributions in (44) are due to the $\operatorname{Re} B_{k_{i} k_{i}}^{R}(0)$ and $\operatorname{Im} \Gamma_{k_{i} k_{i}}(0)$ for all values of the incoming energy $\epsilon_{i}$ except for $\epsilon_{i} \approx n_{0}-\left|\bar{\epsilon}_{b}^{R}\right|$ where the $\operatorname{Im} B_{k_{i} k_{i}}^{R}(0)$ dominates. Thus it is suggestive to define the relative weight $w_{0}(\epsilon)$ of contributions to the transmission coefficient involving transitions between the continuum and the bound state to contributions involving exclusively transitions between continuum states as follows:

$$
w_{0}\left(\epsilon_{i}\right)=\frac{\left|2 \pi \operatorname{Re} B_{k_{i} k_{i}}^{R}(0)\right|}{\left|k_{i}+4 \pi \operatorname{Im} \Gamma_{k_{i} k_{i}}(0)\right|} .
$$

In Fig. 3 we plot for illustration $w_{0}$ as a function of the incoming energy $\epsilon_{i}$ for two different values of the coupling $g_{0}$. We see that the processes involving $\mathrm{c}-\mathrm{c}$ transitions dominate

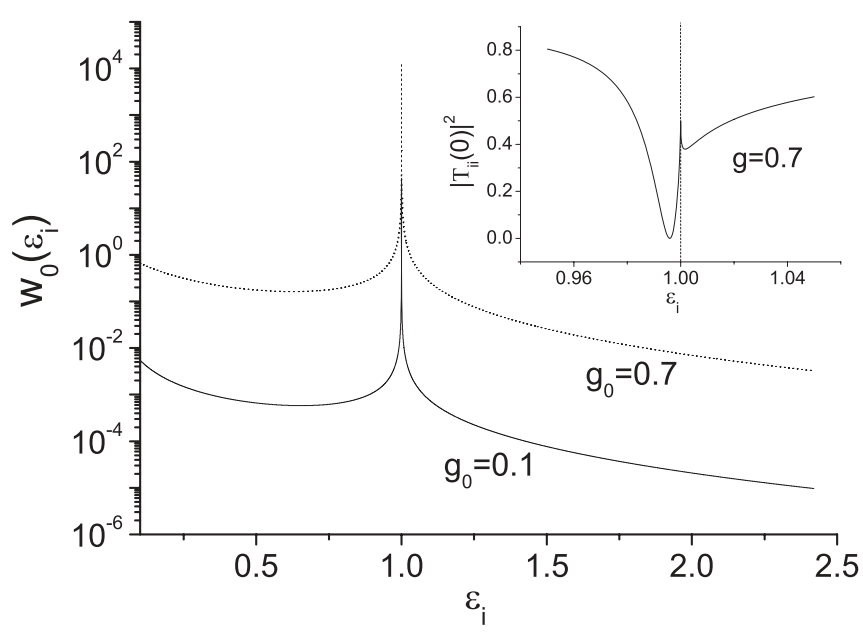

FIG. 3. Relative weight $w_{0}$ of contribution of subprocesses involving continuum-bound state transitions to those involving exclusively continuum-continuum transitions to the elastic part of the transmission coefficient as a function of the incoming energy. Two values of the coupling $g_{0}$ are used: 0.1 (solid line) and 0.7 (dotted line). The inset displays the elastic part of the transmission coefficient in an energy domain around $\epsilon_{i}=1$ for $g_{0}=0.7$.

for the complete energy regime except of a small region around the integer value $\epsilon_{i}=1$.

Existence of a single transmission zero. The dramatic increase of the amplitude of the subprocesses involving transitions between continuum and the bound state around the integer value 1 of the incoming energy has important consequences on the behavior of the transmission coefficient with varying energy (see the inset in Fig. 3). To clarify this let us analyze the elastic channel in more detail. In Eq. (44) the first term corresponds to free transmission, the second to subprocesses with two transitions involving one c-b and one b-c transition, while the third term involves two c-c transitions. The numerator of the second term is of order $O\left(g_{0}^{3}\right)$ while the third term is always of the order of $O\left(g_{0}^{2}\right)$ [25]. The most interesting behavior is associated with the denominator of the second term. As already discussed, the imaginary part $\eta_{0}^{R}$ is of order $O\left(g_{0}^{3}\right)$ [since it does not occur for subprocesses up to $\left.O\left(g_{0}^{2}\right)\right]$. Therefore, whenever $\tilde{\epsilon}_{i}^{R}=n_{0}$ the second term becomes of order one $\left[O\left(g_{0}^{0}\right)\right]$ and dominates. In this case the exact value of $\eta_{0}^{R}$ is crucial since the second term becomes proportional to $1 / \eta_{0}^{R}$. Within our approach it can be determined by going to higher-order terms containing more than two transitions involving the bound state. Since the number of transitions between continuum and bound state is always even, the next-to-leading order contains necessarily four such transitions. The calculation of the contribution to the $S$-matrix amplitude of diagrams involving four transitions between continuum and the bound state is straightforward but lengthy and is not presented here. The final result up to the order $O\left(g_{0}^{4}\right)$ is obtained through the summation of terms originating from diagrams with four and six transitions in total as illustrated in Fig. 4 [26]. The interested reader can find a short description of the calculation in Appendix A.

In the limit $g_{0} / k_{i}<1$, it is straightforward to study the behavior of the transmission coefficient (44) in the case $\tilde{\epsilon}_{i}^{R}=$ $n_{0}$ which also depends on the value of $n_{0}$. Therefore, let us first 


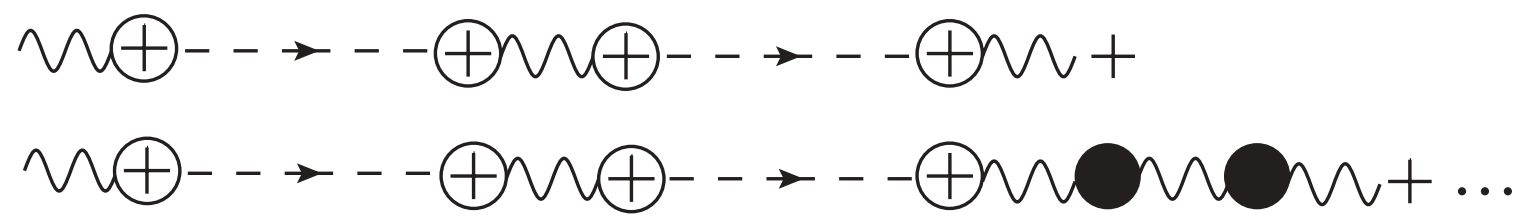

FIG. 4. Diagrams contributing up to the order $O\left(g_{0}^{4}\right)$ to $\eta_{0}^{R}$ and to the shift in $\tilde{\epsilon}_{i}^{R}$. The dots indicate that a sum over all allowed permutations in the 6-transition diagrams must be performed.

investigate the case $n_{0}=1$ for effective incoming energies $0<$ $\tilde{\epsilon}_{i}^{R} \leqslant 1$. When $1-\tilde{\epsilon}_{i}^{R}=O\left(g_{0}\right) \gg \eta_{0}^{R}(1)$ both the imaginary part of the denominator as well as the normalization factor in the second term of Eq. (44) can be neglected and the elastic part of the transmission is well described by the formula

$$
T_{i i}(0) \approx 1-\frac{2 \pi i}{k_{i}} \sum_{n_{0}= \pm 1} \frac{B_{k_{i} b}\left(n_{0}\right) B_{b k_{i}}\left(-n_{0}\right)}{\epsilon_{i}-n_{0}}+\frac{4 \pi}{k_{i}} \operatorname{Im} \Gamma_{k_{i} k_{i}}(0) .
$$

When $1-\tilde{\epsilon}_{i}^{R}=O\left(g_{0}^{2}\right)$, the contribution of the real part of the second term increases and, being negative, leads to a decrease of the transmission amplitude. The effect is maximized when $1-\tilde{\epsilon}_{i}^{R}$ becomes of the order of $O\left(g_{0}^{4}\right)$. Then the denominator of the second term is controlled, almost exclusively, by $\eta_{0}^{R}$, which is of the same order of magnitude as the numerator $B_{k_{i} b}(1) B_{b k_{i}}(-1) \approx O\left(g_{0}^{3}\right)$. Thus, in a narrow energy region around $\epsilon_{i} \approx 1$ the term describing transitions between continuum and bound state dominates (in the considered order of perturbation theory) driving the elastic transmission to zero: $T_{i i}(0) \approx 0$ (for more details see Appendix A). Following a similar reasoning one can show that also the inelastic transmission as well as the reflection amplitude become zero in the considered case while the elastic reflection approaches one.

When the incoming energy increases beyond the position of the transmission zero the expression (45) for the elastic transmission amplitude is again valid. However, the value of the transmission coefficient will slightly decrease due to the fact that the imaginary part of $\Gamma_{k_{i}} k_{i}(0)$ increases with increasing energy and contributes negatively to the transmission amplitude. When the incoming energy is near an integer value $n_{0}>1$, the transmission profile considerably differs from the one around $n_{0}=1$. This is due to the following facts: First, the coefficient $B_{k_{i} b}\left(n_{0}\right) B_{b k_{i}}\left(-n_{0}\right)$ is exponentially suppressed when $n_{0}$ increases and, consequently, the numerator of the second term in Eq. (44) becomes of higher and higher order in $g_{0}$. Second, $\eta_{0}^{R}\left(n_{0}\right)$ is always of order $O\left(g_{0}^{3}\right)$ almost independently of the definite value of $n_{0}$. Therefore, the second term is always suppressed for $n_{0}>1$ with respect to the other two terms. This is an issue which has not been resolved in previous literature. In Ref. [12] it is mentioned that the residues of the higher resonances considered as poles of the transmission amplitude in the complex energy plane decrease with increasing energy. However, no specific explanation has been provided.

Location of transmission zero. As already discussed, due to the higher-order corrections, the transmission zero does not occur for $\epsilon_{i}=1$ but it is slightly shifted to lower values of the incoming energy. In fact, the exact location of the transmission zero is determined by the effective energy $\tilde{\epsilon}_{i}^{R}$. Within our treatment it is straightforward to obtain not only $\eta_{0}^{R}$ but also the shift in the effective energy $\tilde{\epsilon}_{i}^{R}$ as well as the normalization factor $Z_{0}$ appearing in Eq. (44):

$$
\begin{aligned}
\eta_{0}^{R}\left(n_{0}\right) & \approx \beta\left(n_{0}\right)\left[1+\gamma_{0}\left(n_{0}\right)\right], \\
\tilde{\epsilon}_{i}^{R}\left(n_{0}\right) & \approx \epsilon_{i}+\frac{1}{8} g_{0}^{2}+\alpha\left(n_{0}\right), \\
Z_{0} & \approx 1-\frac{2 \pi i}{k_{i}}\left[4 i \operatorname{Im} \Gamma_{k_{i} k_{i}}(0)-\sum_{l \neq n_{0}} \frac{B_{k_{i} b}(-l) B_{b k_{i}}(l)}{\tilde{\epsilon}_{i}^{R}\left(n_{0}\right)-l+i \eta_{0}^{R}\left(n_{0}\right)}\right],
\end{aligned}
$$

with

$$
\begin{aligned}
& \beta\left(n_{0}\right)=\sum_{l} \frac{2 \pi}{\sqrt{k_{i}^{2}+2 l}} B_{k_{i} b}\left(n_{0}+l\right) B_{b k_{i}}\left(-n_{0}-l\right), \\
& \gamma_{0}\left(n_{0}\right)=\frac{4 \pi}{k_{i}} \operatorname{Im} \Gamma_{k_{i} k_{i}}(0), \\
& \alpha\left(n_{0}\right)=2 \sum_{l} \operatorname{Pr} \int_{0}^{\infty} d k \frac{B_{k b}\left(n_{0}+l\right) B_{b k}\left(-l-n_{0}\right)}{\epsilon_{k}-\epsilon_{i}-l} .
\end{aligned}
$$

More details of the calculation of these quantities are given in Appendix A. It is important to notice here that the effect of the higher-order corrections leading to the shift of the effective incoming energy and to the appearance of the imaginary part $\eta_{0}^{R}$ in the denominator of the second term in Eq. (44) starts to show up in the immediate neighborhood of the transmission zero [i.e., when $\left.1-\tilde{\epsilon}_{i}^{R}=O\left(g_{0}^{2}\right)\right]$. In the literature the transmission zero is associated with a Fano resonance [11]. Its exact position however has not been obtained from first principles. In Ref. [12] it is estimated by a continued-fractions approach. Within our treatment it is in principle possible [see Eq. (46) and Appendix A] to obtain the position of the transmission zero to arbitrary accuracy taking into account terms with increasing order in the proposed perturbation scheme. In addition our treatment provides a physical picture for the origin of this shift: it is attributed to a hierarchical sequence of subprocesses involving repetitive transitions of the incoming particle from the continuum to the bound state and back. These can be identified with a virtual "multiphoton" exchange of the propagating particle with the driving potential. Subprocesses with increasing number of transitions become more and more suppressed.

Further features of transmission coefficient. In addition to the above analyzed transmission zero there occur pronounced local peaks in the transmission coefficient with increasing incoming energy. A typical structure is shown in Fig. 5(a) where a zoom in the region around the zero of the transmission coefficient in the elastic channel is performed. This structure is attributed to the competition between the real part of the second 

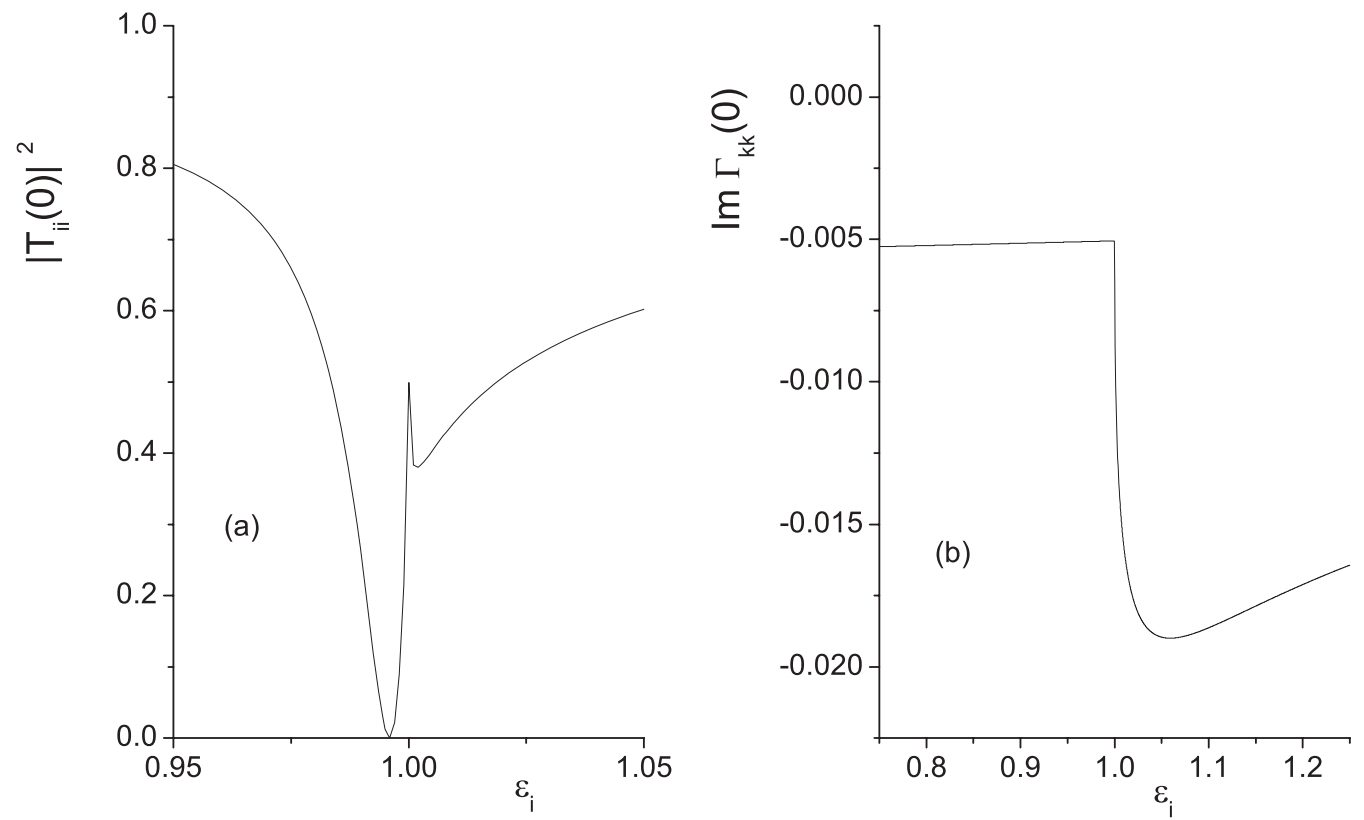

FIG. 5. (a) Elastic part of the transmission coefficient as a function of incoming energy for $g_{0}=0.7$ in the region around the transmission zero and (b) $\operatorname{Im} \Gamma_{k_{i} k_{i}}(0)$ as a function of incoming energy in the same region.

term in Eq. (44) and the contribution proportional to $\operatorname{Im} \Gamma_{k_{i} k_{i}}(0)$ contained in the third term. The latter possesses a cusp at $\epsilon_{i}=1$ [see Appendix B and Fig. 5(b)] and contributes destructively (negative sign) in the transmission amplitude while the former decreases rapidly as the incoming energy increases beyond the transmission-zero value. Depending on the position of the transmission zero with respect to the value 1 where the discontinuity of $\operatorname{Im} \Gamma_{k_{i} k_{i}}(0)$ is located, this competition may lead to a pronounced effect (the observed peak structure) or not. For the occurrence of the peak structure, the distance between transmission zero and $\epsilon_{i}=1$ should be at least of the order of $g_{0}^{2}$. It must be noticed that the discontinuity in $\operatorname{Im} \Gamma_{k_{i} k_{i}}(0)$ is a consequence of integrated poles in the corresponding $S$-matrix amplitudes.

\section{CONCLUDING REMARKS}

In the present work we have developed a perturbative scheme for the calculation of the $S$ matrix for a scattering problem involving a time-dependent external potential. The approximation scheme we propose can, in fact, be used to probe the dynamics of any system (even a completely bound one) in which an external parameter changes very slowly or very fast. The quintessence of the approach is based on the fact that, when the first- or the second-quantized dynamics of the driven system is expressed in terms of the basis of the instantaneous eigenstates, an effective Hamiltonian appears [22]. This Hamiltonian contains the nondiagonal geometric phases that express the amplitudes for transitions between different states and which can be treated as small perturbations. In this framework the analytic knowledge of the complete set of the temporary eigenstates is not of vital importance since the transitions between very distant states can be considered as strongly suppressed.
We have used this approach to describe the transmission through a $\delta$ potential with harmonically oscillating strength as a first simple application. Here, the temporary spectrum may contain also a single bound state. An interesting feature of the developed perturbation method is that the transitions between a continuum state of the temporary spectrum and the bound state depend on a different power of the coupling of the potential than the transitions between two continuum states. Therefore, the perturbative approach has to be applied with special care in order to avoid artifacts. The proposed scheme has several advantages providing a tool for a detailed study of the scattering process based on the intuitive particle picture instead of the usual wave picture which is implied by methods focusing on the wave-function calculation. These advantages are here demonstrated by establishing a systematic procedure for the exact calculation of the transmission-zero properties associated with the appearance of a Fano resonance in the considered system. Within the proposed scheme, it is possible to show that the transition amplitudes of the inelastic channels decrease exponentially with increasing order in the Floquet expansion (real "multiphoton" exchange). This fact explains the appearance of only one resonant energy in the transmission coefficient. Furthermore, our approach reveals the significance of virtual "multiphoton" exchange processes which are responsible for a slight shift of the location of the transmission zero. Finally, the developed method enables also the detailed analysis of local peak structures occurring in the dependence of the transmission coefficient on the incoming energy which cannot be attributed to resonances (poles of the transmission amplitudes) and are usually not investigated in the literature.

A disadvantage of the proposed method is that it is technically more demanding than other existing methods, mentioned above, possessing several anomalies similar to those of quantum field perturbation theory. Therefore, a 
computational strategy has to be developed enabling the application of the method to arbitrary-shaped potentials, a task which is left for the future. Here, we have merely concentrated on the theory development and the introduction of the basic concepts of this approach. The occurring technical complications are balanced by the deeper insight gained for the considered physical problem opening the perspective of a systematic decomposition of the time-dependent scattering process in fundamental subprocesses building up the skeleton of transport in driven quantum systems.

\section{ACKNOWLEDGMENTS}

This research has been cofinanced by the European Union (European Social Fund ESF) and Greek national funds through the Operational Program "Education and Lifelong Learning" of the National Strategic Reference Framework (NSRF) - Research Funding Program: Heracleitus II. Investing in knowledge society through the European Social Fund. Further financial support by the Greek Scholarship Foundation IKY in the framework of an exchange program with Germany (IKYDA) is also acknowledged.

\section{APPENDIX A}

In this appendix we shall briefly present the calculations that lead to the corrected values appearing in Eq. (39) of the text. To begin with, we note that the need to consider higherorder corrections can be understood in terms of the following schematic expansion:

$$
\frac{B^{2}}{\left(\tilde{\varepsilon}_{i}+\delta \varepsilon\right)-n_{0}+i(\eta+\delta \eta)} \approx \frac{B^{2}}{\tilde{\varepsilon}_{i}-n_{0}+i \eta}-\frac{B^{2} \delta \varepsilon}{\left(\tilde{\varepsilon}_{i}-n_{0}+i \eta\right)^{2}}-i \frac{B^{2} \delta \eta}{\left(\tilde{\varepsilon}_{i}-n_{0}+i \eta\right)^{2}}+\cdots .
$$

It is obvious that, when $\tilde{\varepsilon}_{\iota} \approx n_{0}$, the corrections $\delta \varepsilon$ and $\delta \eta$ must be taken into account. The first process that contains a double pole term involves in total 4 transitions, all of b-c or c-b type:

$$
\begin{aligned}
S_{f i}^{(4 t, 0 c, 4 b)} & \approx-4 \pi i \sum_{n, n_{1}, n_{2}, n_{3}} \frac{B_{k_{f} b}\left(n-n_{3}\right)}{\tilde{\varepsilon}_{i}+n_{3}+i \eta}\left[\int_{0}^{\infty} d k \frac{B_{b k}\left(n_{3}-n_{2}\right) B_{k b}\left(n_{2}-n_{1}\right)}{\varepsilon_{i}-\varepsilon_{k}+n_{2}+i \eta}\right] \frac{B_{b k_{i}}\left(n_{1}\right)}{\tilde{\varepsilon}_{i}+n_{1}+i \eta} \\
& \approx n_{1}=n_{3} \rightarrow-n_{0} \\
& -2 \pi i \sum_{n, n_{0}} \frac{B_{k_{f} b}\left(n+n_{0}\right) B_{b k_{i}}\left(-n_{0}\right)}{\left(\tilde{\varepsilon}_{i}-n_{0}+i \eta\right)^{2}}\left[-\alpha\left(n_{0}\right)-i \beta\left(n_{0}\right)\right] .
\end{aligned}
$$

In the last expression we wrote

$$
\alpha\left(n_{0}, \varepsilon_{i}\right) \equiv 2 \sum_{l} \operatorname{Pr} \int_{0}^{\infty} d k \frac{B_{k b}\left(n_{0}+l\right) B_{b k}\left(-l-n_{0}\right)}{\varepsilon_{k}-\varepsilon_{i}-l}
$$

and

$$
\begin{aligned}
\beta\left(n_{0}, \varepsilon_{i}\right) & \equiv \sum_{l} \frac{2 \pi}{k_{l}} B_{k_{l} b}\left(n_{0}+l\right) B_{b k_{l}}\left(-l-n_{0}\right), \\
\left(k_{l}\right. & \left.=\sqrt{k_{i}^{2}+2 l}\right) .
\end{aligned}
$$

Comparing Eq. (A2) with the expansion (A1) we get the corrected quantities appearing in the text:

$$
\begin{gathered}
\tilde{\varepsilon}_{i}^{R}\left(n_{0}\right) \approx \varepsilon_{i}+\frac{1}{8} g^{2}+\alpha\left(n_{0}, \varepsilon_{i}\right), \\
\eta_{n_{0}}^{R} \approx \beta\left(n_{0}, \varepsilon_{i}\right) .
\end{gathered}
$$

Some comments are in order at this point. The function $\alpha$ corrects the pole position:

$$
\varepsilon_{i}=n_{0}-\frac{1}{8} g^{2}-\alpha\left(n_{0}, \varepsilon_{i}\right) .
$$

Its leading behavior in the limit $g_{0} / k_{i}<1$ is obtained for $n_{0}+l= \pm 1$ and it is almost independent of $n_{0}$, having the form

$$
\alpha \approx-g^{2} \alpha_{1}-g^{4} \alpha_{2}, \quad \alpha_{1,2}>0 .
$$

The nonvanishing value of $\beta$ [see Eq. (A4)] drives to finite values all the terms in the perturbative calculation of the
$S$ matrix. Similarly to $\alpha$, its leading behavior, being of order $O\left(g_{0}^{3}\right)$, is obtained for the combination $n_{0}+l= \pm 1$ and it is almost independent of $n_{0}$. This behavior is responsible for the zero of the elastic transmission amplitude: for $\varepsilon_{i} \approx 1-\frac{1}{8} g^{2}-\alpha$, the amplitude $\frac{2 \pi i}{k_{i}} B_{k_{i} k_{i}}^{R}(0)$ dominates and, being real, positive, and of order $O(1)$ cancels the free transmission contribution driving the total amplitude to zero. For $\varepsilon_{i} \approx n_{0}>1$ the phenomenon is suppressed since $B_{k_{i} k_{i}}^{R}(0) \sim O\left(g_{0}^{2\left(n_{0}-1\right)}\right)$.

For the quantitative analysis of the scattering process, in the energy region in which the "pole" behaviour is visible, one needs a more exact value of $\beta$ and this calls for corrections coming from higher-order processes. The 5-transitions amplitude that contains $4 \mathrm{~b}-\mathrm{c}$ or $\mathrm{c}-\mathrm{b}$ transitions and one $\mathrm{c}-\mathrm{c}$ transition, gives a higher-order correction of the pole position and we shall not discuss it here. On the other hand the 6-transitions amplitude with $4 \mathrm{~b}-\mathrm{c}$ or c-b and two c-c transitions contributes to $\eta$; that is, to the imaginary part of $B_{k_{f} k_{i}}^{R}(n)$ :

$$
\begin{aligned}
S_{f i}^{(6 t, 2 c, 4 b) \approx} & -2 \pi i \sum_{n_{0}} \frac{B_{k_{i} b}\left(n+n_{0}\right) B_{b k_{i}}\left(-n_{0}\right)}{\left(\tilde{\varepsilon}_{i}-n_{0}+i \eta\right)^{2}} \\
& \times\left[-i \gamma_{n}\left(n_{0}\right) \beta\left(n_{0}\right)\right],
\end{aligned}
$$

where

$$
\gamma_{n}\left(n_{0}\right) \equiv \frac{2 \pi}{k_{i}} \operatorname{Im}\left[\Gamma_{k_{i} k_{i}}(0)+\frac{B_{k_{i} b}\left(n_{0}\right)}{B_{k_{f} b}\left(n+n_{0}\right)} \Gamma_{k_{f} k_{i}}(n)\right] .
$$


Combining the above results with Eq. (A2) we find

$$
\eta_{n}^{R} \approx \beta\left(n_{0}\right)\left[1+\gamma_{n}\left(n_{0}\right)\right]
$$

For the elastic channel $n=0$ the last result is apparent in Eq. (46). When the incoming energy is at the realm of the "pole," higher-order corrections of the pole residue, coming from first-order poles in the 3-transitions amplitude with two $\mathrm{b}-\mathrm{c}$ or $\mathrm{c}-\mathrm{b}$ and one $\mathrm{c}-\mathrm{c}$ transitions and in the 4-transitions amplitude with $2 \mathrm{~b}-\mathrm{c}$ or $\mathrm{c}-\mathrm{b}$ and $2 \mathrm{c}-\mathrm{c}$ transitions, must be taken into account:

$$
\begin{aligned}
& S_{f i}^{(3 t, 1 c, 2 b)}+S_{f i}^{(4 t, 2 c, 2 b)} \\
& \quad \approx-2 \pi i \sum_{n_{0}} \frac{B_{k_{f} b}\left(n+n_{0}\right) B_{b k_{i}}\left(-n_{0}\right)}{\tilde{\varepsilon}_{i}-n_{0}+i \eta}\left(Z_{n}-1\right)
\end{aligned}
$$

where

$$
\begin{aligned}
Z_{n} \approx 1 & -\frac{2 \pi i}{k_{i}}\left[2 \Gamma_{k_{i} k_{i}}(0)+\frac{B_{k_{i} b}\left(n_{0}\right)}{B_{k_{f} b}\left(n+n_{0}\right)}\left(2 \Gamma_{k_{f} k_{i}}(n)-A_{k_{f} k_{i}}(n)\right)\right. \\
& \left.-\sum_{l \neq-n_{0}} \frac{B_{k_{f} b}(n-l) B_{b k_{i}}(l)}{\tilde{\varepsilon}_{i}+l+i \eta} \frac{B_{k_{i} b}\left(n_{0}\right)}{B_{k_{f} b}\left(n+n_{0}\right)}\right] . \quad \text { (A13) }
\end{aligned}
$$

Combining the results of Eqs. (A5), (A11), and (A13) we find the normalized contribution of the $\mathrm{b}-\mathrm{c}$ or $\mathrm{c}-\mathrm{b}$ transitions to the scattering process as given in Eq. (39):

$$
B_{k_{f} k_{i}}^{R}(n)=\sum_{n_{0}=-\infty}^{\infty} \frac{B_{k_{f} b}\left(n+n_{0}\right) B_{b k_{i}}\left(-n_{0}\right)}{\varepsilon_{i}^{R}\left(n_{0}\right)-n_{0}+i \eta_{n}^{R}\left(n_{0}\right)} Z_{n} .
$$

The above formulas capture the nontrivial behaviour of the scattering amplitude in the vicinity of a "pole." For example, the zero of the elastic transmission amplitude in the region $\left|1-\varepsilon_{i}^{R}\right| \ll \eta_{0}^{R}$ is easily verified:

$$
\begin{aligned}
T_{i i}(0) \approx & 1-\frac{2 \pi i}{k_{i}} \sum_{l \neq 1} \frac{B_{k_{i} b}(l) B_{b k_{i}}(-l)}{\varepsilon_{i}-l}-\frac{2 \pi i}{k_{i}} \frac{B_{k_{i} b}(1) B_{b k_{i}}(-1)}{i \eta^{R}(1)} Z_{0}+\frac{4 \pi}{k_{i}} \operatorname{Im} \Gamma_{k_{i} k_{i}}(0) \\
\approx & 1-\frac{2 \pi i}{k_{i}} \sum_{l \neq 1} \frac{B_{k_{i} b}(l) B_{b k_{i}}(-l)}{\varepsilon_{i}-l}-\frac{2 \pi i}{k_{i}} \frac{B_{k_{i} b}(1) B_{b k_{i}}(-1)}{\frac{2 \pi i}{k_{i}} B_{k_{i} b}(1) B_{b k_{i}}(-1)\left[1+\frac{4 \pi}{k_{i}} \operatorname{Im} \Gamma_{k_{i} k_{i}}(0)\right]} \\
& \times\left[1+\frac{8 \pi}{k_{i}} \operatorname{Im} \Gamma_{k_{i} k_{i}}(0)-\frac{2 \pi i}{k_{i}} \sum_{l \neq 1} \frac{B_{k_{i} b}(l) B_{b k_{i}}(-l)}{\varepsilon_{i}-l}\right]+\frac{4 \pi}{k_{i}} \operatorname{Im} \Gamma_{k_{i} k_{i}}(0) \\
\approx & 0 .
\end{aligned}
$$

It is not difficult to show that in the same energy region and at the same order the inelastic amplitudes also vanish:

$$
T_{f i}(n)=R_{f i}(n) \approx-\frac{k_{i}}{\left|k_{f}\right|} \frac{B_{k_{f} b}(n+1)}{B_{k_{i} b}(1)}\left[1+\frac{2 \pi}{k_{i}} \operatorname{Im} \Gamma_{k_{i} k_{i}}(0)\right] .
$$

The term $n=1$ does not contribute since $B(2)=0$. For $n>1$ the coefficients

$$
R(n)=T(n) \equiv \frac{\left|k_{f}\right|}{k_{i}}\left|T_{f i}(n)\right|^{2}
$$

are negligible in comparison to $R(0) \approx 1$.

\section{APPENDIX B}

In Appendix A (and in the main text) it is extensively used that the imaginary part of the amplitude

$$
\Gamma_{k_{i} k_{i}}(0)=\int_{0}^{\infty} d k \sum_{l=-\infty}^{\infty} \frac{A_{k_{i} k}(-l) A_{k k_{i}}(l)}{\varepsilon_{i}-\varepsilon_{k}+l+i \eta}
$$

dominates over its real part. Besides the numerical confirmation, we present in this appendix a direct proof of this statement. Using the expression (27) it is straightforward to obtain

$$
\begin{aligned}
\operatorname{Im} \Gamma_{k_{i} k_{i}}(0)= & -\frac{k_{i}^{2}}{4 \pi} \sum_{\substack{l \neq 0 \\
k_{l}^{2}=k_{i}^{2}+2 l \geqslant 0}} \frac{k_{l}}{l^{2}} \frac{1}{\left(k_{i}+k_{l}\right)^{2}} \\
& \times\left|q_{\bar{k}_{l}}(|l|)-(-1)^{l} q_{\bar{k}_{i}}(|l|)\right|^{2},
\end{aligned}
$$

with

$$
q_{\bar{k}}(|l|)=\left(\sqrt{\bar{k}^{2}+1}-\bar{k}\right)^{|l|}, \quad \bar{k}=k / g_{0} .
$$

Observing that: $q_{\bar{k}_{l}}(|l|) \sim\left(g_{0} / k_{l}\right)^{|l|}$ we immediately find $\operatorname{Im} \Gamma_{k_{i} k_{i}}(0) \sim\left(g_{0} / k_{i}\right)^{2}$. This estimation is valid if $k_{l}^{2}=k_{i}^{2}+$ $2 l>k_{i}^{2}$ (i.e., if $l>0$ ). However, negative values of $l$ are permitted if the incoming energy is large enough. Thus, every time the incoming energy increases by an integer value, the possibility of an additional negative value of $l$ occurs. For example, if $k_{i}^{2} / 2=1+O\left(g_{0}^{2}\right)$ the value $l=-1$ is permitted and, in fact, it gives the dominant contribution to the sum (B2) since $k_{l} \approx O\left(g_{0}\right)$ and $\operatorname{Im} \Gamma_{k_{i} k_{i}}(0) \approx O\left(g_{0}\right)$. This is the technical explanation for the cusp appearing in Fig. 5 of the text. The real part of the amplitude can also be calculated analytically. Using the abbreviations $\lambda_{\bar{k}}=\sqrt{\bar{k}^{2}+1}-\bar{k}, \kappa_{\bar{k}}=\sqrt{\bar{k}^{2}+1}, \sum_{l^{\prime}}(\cdots)=$ $\sum_{k_{l}^{2}=k_{i}^{2}+2 l \geqslant 0}(\cdots)$ we find that

$$
\operatorname{Re} \Gamma_{k_{i} k_{i}}(0)=I_{1}+I_{2} \text {. }
$$


The first term on the rhs of the last equation is

$$
I_{1}=\frac{k_{i}^{2}}{16 \pi^{2}} \sum_{l^{\prime}} \frac{k_{l}^{2} \ln k_{l}^{2}}{l^{2}} \frac{\left(A_{1} B_{1}\right)}{g_{0}^{3}}
$$

where

$$
A_{1}=q_{\bar{k}_{l}}(|l|)\left[\frac{1}{\bar{k}_{l}\left(\bar{k}_{l}-\bar{k}_{i}\right)}+\frac{(-1)^{l}}{\bar{k}_{l}\left(\bar{k}_{l}+\bar{k}_{i}\right)}\right]-\frac{2}{\bar{k}_{l}^{2}-\bar{k}_{i}^{2}} q_{\bar{k}_{i}}(|l|),
$$

and

$$
\begin{aligned}
B_{1}= & q_{\bar{k}_{l}}(|l|-1)\left(\lambda_{\bar{k}_{l}}^{2}-1\right)\left[\frac{1}{\bar{k}_{l}\left(\bar{k}_{l}-\bar{k}_{i}\right)}+\frac{(-1)^{l-1}}{\bar{k}_{l}\left(\bar{k}_{l}+\bar{k}_{i}\right)}\right] \\
& -\frac{2}{\bar{k}_{l}^{2}-\bar{k}_{i}^{2}} q_{\bar{k}_{i}}(|l|-1)\left(\lambda_{\bar{k}_{i}}^{2}-1\right) .
\end{aligned}
$$

The second term has the form

$$
I_{2}=-\frac{k_{i}^{4} \ln k_{i}^{2}}{4 \pi^{2}} \sum_{l^{\prime}} \frac{1}{l^{2}} \frac{\left(A_{2} B_{2}\right)}{g_{0}^{3}},
$$

with

$$
\begin{aligned}
A_{2}= & \frac{1}{2} \frac{q_{\bar{k}_{i}}(|l|+1)}{\kappa_{\bar{k}_{i}}^{2} \bar{k}_{i}}-\frac{|l|+1}{2} \frac{q_{\bar{k}_{i}}(|l|)}{\kappa_{\bar{k}_{i}} \bar{k}_{i}}-\frac{\lambda \bar{k}_{\bar{k}_{i}}^{|l|+1}}{\kappa_{\bar{k}_{i}}^{2} \bar{k}_{i}} \\
& -\frac{1}{4} \frac{q_{\bar{k}_{i}}(|l|)}{\kappa_{\bar{k}_{i}}^{2} \bar{k}_{i}}\left(\lambda_{\bar{k}_{i}}-\bar{k}_{i}\right)+\frac{(-1)^{l}}{4} \frac{q_{\bar{k}_{i}}(|l|)}{\bar{k}_{i}^{2}}
\end{aligned}
$$

and

$$
\begin{aligned}
B_{2}= & \frac{q_{\bar{k}_{i}}(|l|)}{\kappa_{\bar{k}_{i}}^{2} \bar{k}_{i}}\left(\lambda_{\bar{k}_{i}}^{2}-1\right)-|l| \frac{q_{\bar{k}_{i}}(|l|-1)}{\kappa_{\bar{k}_{i}} \bar{k}_{i}}\left(\lambda_{\bar{k}_{i}}^{2}-1\right)-4 \frac{\lambda_{\bar{k}_{i}}^{|l|+2}}{\kappa_{\bar{k}_{i}}^{2} \bar{k}_{i}} \\
& -\frac{1}{2} \frac{q_{\bar{k}_{i}}(|l|-1)}{\kappa_{\bar{k}_{i}}^{2} \bar{k}_{i}}\left(\lambda_{\bar{k}_{i}}^{2}-1\right)\left(\lambda_{\bar{k}_{i}}-\bar{k}_{i}\right) \\
& +\frac{(-1)^{l-1}}{2} \frac{q_{\bar{k}_{i}}(|l|-1)}{\bar{k}_{i}^{2}}\left(\lambda_{\bar{k}_{i}}^{2}-1\right) .
\end{aligned}
$$

Observing that $\lambda_{\bar{k}} \sim g_{0}, \kappa_{\bar{k}} \sim 1 / g_{0}$ we immediately show that $A_{1} \underset{l=1}{\sim} g_{0}^{3}, B_{1} \underset{l=1}{\sim} g_{0}^{4}$ and $A_{2} \underset{l=1}{\sim} g_{0}^{3}, B_{2} \underset{l=1}{\sim} g_{0}^{4}$ and, consequently, that $\operatorname{Re} \Gamma_{k_{i} k_{i}}(0) \sim g_{0}^{4}$.

The case $k_{i}^{2}+2 l<0$ can be treated by using the above formulas and making the change $k_{l} \rightarrow-i\left|k_{l}\right|$ in the expressions for $\operatorname{Re} \Gamma_{k_{i} k_{i}}(0)+i \operatorname{Im} \Gamma_{k_{i} k_{i}}(0)$. The final result is the same.
[1] A. del Campo, G. García-Calderon, and J. G. Muga, Phys. Rep. 476, 1 (2009).

[2] A. O. Caldeira and A. J. Leggett, Ann. Phys. (NY) 149, 374 (1983).

[3] R. Bruinsma and P. Bak, Phys. Rev. Lett. 56, 420 (1986).

[4] M. Büttiker and R. Landauer, Phys. Rev. Lett. 49, 1739 (1982).

[5] E. H. Hauge and J. A. Stovneng, Rev. Mod. Phys. 61, 917 (1989); E. Eisenberg and Y. Ashkenazy, Found. Phys. 27, 191 (1997); A. V. Pimpale, Prog. Quantum Electron. 28, 345 (2004).

[6] M. Switkes, C. M. Marcus, K. Campman, and A. C. Gossard, Science 289, 1905 (1999); S. Kohler, J. Lehmann, and P. Hänggi, Phys. Rep. 406, 379 (2005).

[7] D. A. B. Miller, Opt. Eng. 26, 368 (1987); N. H. Bonadeo et al., Science 282, 1473 (1998); F. H. L. Koppens et al., Nature (London) 442, 766 (2006).

[8] D. Müller, E. A. Cornell, M. Prevedelli, P. D. D. Schwindt, A. Zozulya, and D. Z. Anderson, Opt. Lett. 25, 1382 (2000); T. Schumm, S. Hofferberth, L. M. Andersson, S. Wildermuth, S. Groth, I. Bar-Joseph, J. Schmiedmayer, and P. Krüger, Nature Phys. 1, 57 (2005).

[9] T. Lahaye, J. M. Vogels, K. J. Günter, Z. Wang, J. Dalibard, and D. Guery-Odelin, Phys. Rev. Lett. 93, 093003 (2004); E. Torrontegui, J. Echanobe, A. Ruschhaupt, D. Guery-Odelin, and J. G. Muga, Phys. Rev. A 82, 043420 (2010).

[10] A. Pimpale and M. Razavy, Fortschr. Phys. 39, 85 (1991); E. Cota, J. V. Jose, and F. Rojas, Nanostruct. Mater. 3, 349 (1993); A. V. Pimpale, Prog. Quantum Electron. 28, 345 (2004).

[11] P. F. Bagwell and R. K. Lake, Phys. Rev. B 46, 15329 (1992).

[12] D. F. Martinez and L. E. Reichl, Phys. Rev. B 64, 245315 (2001).

[13] J. Campbell, J. Phys. A 42, 365212 (2009).

[14] M. Moskalets and M. Büttiker, Phys. Rev. B 68, 075303 (2003); S. W. Kim, Int. J. Mod. Phys. B 18, 3071 (2004); C. Benjamin, Eur. Phys. J. B 52, 403 (2006); M. M. Mahmoodian, L. S. Braginsky, and M. V. Entin, Phys. Rev. B 74, 125317 (2006);
M. M. Mahmoodian and M. V. Entin, Europhys. Lett. 77, 67002 (2007); M. M. Makhmudian, M. V. Entin, and L. S. Braginsky, J. Exp. Theor. Phys. 105, 495 (2007); M. Moskalets and M. Büttiker, Phys. Rev. B 75, 035315 (2007).

[15] F. K. Diakonos, P. Kalozoumis, A. I. Karanikas, N. Manifavas, and P. Schmelcher (in preparation).

[16] D. F. Martinez, L. E. Reichl, and G. A. Luna-Acosta, Phys. Rev. B 66, 174306 (2002).

[17] D. J. Tannor, Introduction to Quantum Mechanics, a TimeDependent Perspective (University Science Books, Sausalito, 2007).

[18] M. Moskalets and M. Büttiker, Phys. Rev. B 72, 035324 (2005); 78, 035301 (2008).

[19] H. Kleinert, Path Integrals in Quantum Mechanics, Statistics, Polymer Physics, and Financial Markets (World Scientific, Singapore, 2009).

[20] S. N. Storchak, Phys. Lett. A 161, 397 (1992); V. V. Dodonov, V. I. Manko, and D. E. Nikonov, ibid. 162, 359 (1992); C. Grosche, ibid. 182, 28 (1993); S. Albeverio and S. Mazzuchi, J. Funct. Anal. 238, 471 (2006); D. Laroze, G. Gutierez, R. Rivera, and J. M. Yanez, Phys. Scr. 78, 015009 (2008); S. Pepore and B. Sukbot, Indonesian J. Phys. 21, 47 (2010).

[21] A. Balaz et al., J. Stat. Mech.: Theory Exp. (2011) P03004; (2011) P03005.

[22] K. Fujikawa, Phys. Rev. D 72, 025009 (2005); 77, 045006 (2008).

[23] S. M. Blinder, Phys. Rev. A 37, 973 (1988).

[24] S.-W. V. Chung, M. Moskalets, and P. Samuelsson, Phys. Rev. B 75, 115332 (2007).

[25] For notational simplicity we use the ordering with respect to powers of $g_{0}$ although it is always meant the ordering with respect to powers of $g_{0} / k$.

[26] A careful counting shows that the contribution of diagrams involving 5 transitions to the finite value of $\eta_{0}^{R}$ is of higher order. 